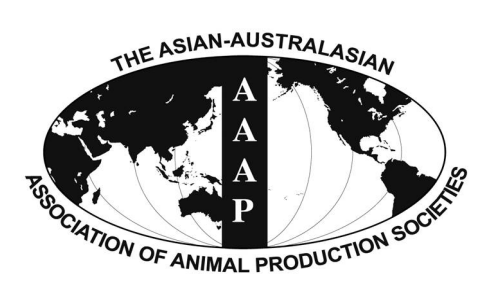

Open Access

Asian Australas. J. Anim. Sci.

Vol. 29, No. 3 : 365-371 March 2016

http://dx.doi.org/10.5713/ajas.15.0626

www.ajas.info

pISSN 1011-2367 elSSN 1976-5517

\title{
The In vitro Effects of Nano-encapsulated Conjugated Linoleic Acid on Stability of Conjugated Linoleic Acid and Fermentation Profiles in the Rumen
}

\author{
Wan Heo ${ }^{\text {a }}$, Eun Tae Kim ${ }^{1, a}$, Sung Do Cho ${ }^{2}$, Jun Ho Kim, Seong Min Kwon, Ha Yeon Jeong', \\ Kwang Seok Ki ${ }^{1}$, Ho Baek Yoon ${ }^{1}$, Young Dae Ahn ${ }^{3}$, Sung Sill Lee ${ }^{4}$, and Young Jun Kim* \\ Department of Food and Biotechnology, Korea University, Sejong 339-700, Korea
}

\begin{abstract}
This study was aimed to evaluate the stability of conjugated linoleic acids (CLAs) by nano-encapsulation against in vitro ruminal biohydrogenation by microbial enzymatic conversion. CLAs (free fatty acid form of CLA [CLA-FFA], nano-encapsulated CLA-FFA, triglyceride form of CLA [CLA-TG], and nano-encapsulated CLA-TG) were used in the in vitro fermentation experiments. When Butyrivibrio fibrisolvens (B. fibrisolvens) was incubated with CLA-FFAs, the concentrations of cis-9, trans-11 CLA and vaccenic acid (VA) slightly was decreased and increased by nano-encapsulation, respectively. When $B$. fibrisolvens was incubated with CLA-TG, the concentrations of cis-9, trans-11 CLA and VA decreased, but these were increased when B. fibrisolvens was incubated with nanoencapsulated CLA-TG. The nano-encapsulation was more effective against the in vitro biohydrogenation activity of $B$.fibrisolvens incubated with CLA-FFA than with CLA-TG. In the in vitro ruminal incubation test, the total gas production and concentration of total volatile fatty acids incubated with nano-encapsulated CLA-FFA and CLA-TG were increased significantly after $24 \mathrm{~h}$ incubation $(\mathrm{p}<0.05)$. Nano-encapsulated CLA-FFA might, thus, improve the ruminal fermentation characteristics without adverse effects on the incubation process. In addition, nano-encapsulated CLA-FFA increased the population of Fibrobacter succinogenes and decreased the population of $B$. fibrisolvens population. These results indicate that nano-encapsulation could be applied to enhance CLA levels in ruminants by increasing the stability of CLA without causing adverse effects on ruminal fermentation. (Key Words: Biohydrogenation, Conjugated Linoleic Acid, Nano-encapsulation, Rumen Fermentation)
\end{abstract}

\section{INTRODUCTION}

An increased interest in conjugated linoleic acid (CLA) in the dairy industry is attributed to its positive role in cancer prevention, decreased risk of atherosclerosis, and improved immune response (Cook et al., 2003; Kathirvelan

\footnotetext{
* Corresponding Author: Young Jun Kim. Tel: +82-44-860-1435, Fax:+82-44-865-0220, E-mail: yk46@korea.ac.kr

${ }^{1}$ National Institute of Animal Science, RDA, Cheonan 331-808, Korea.

${ }^{2}$ Policy Research and Planning Team, Korea Institute for Advancement of Technology, Seoul 135-513, Korea.

${ }^{3}$ Cheongwon Green Land, Cheongju 363-820, Korea.

${ }^{4}$ Division of Applied Life Science (BK2 $1^{+}$, IALS), Gyeongsang

National University, Jinju 660-701, Korea.

${ }^{\mathrm{a}}$ These authors contributed equally to this work.

Submitted Jul. 27, 2015; Revised Aug. 25, 2015; Accepted Sept. 9, 2015
}

et al., 2008; Dilzer and Park, 2012). The predominant natural CLA isomer is cis-9, trans-11 CLA, which account for $80 \%$ to $90 \%$ of the total CLA in dairy products (Parodi, 2003; Crumb, 2011). The activity of a variety of ruminal microbes plays a key role in the conversion of dietary polyunsaturated fatty acids (PUFAs) to saturated fatty acids (SFAs) through the biohydrogenation pathways in the rumen. Linoleic acid (LA), the most common natural source of CLA, is converted to CLA and/or rapidly disappears in the rumen via the activity of ruminal bacteria and enzymes through the metabolic processes (Chin et al., 1992; Shantha et al., 1994). Alternatively, CLA occurs as an intermediate during the biohydrogenation of LA in the rumen by Butyrivibrio fibrisolvens (B. fibrisolvens) (Kritchevsky, 2000). The high degree of accumulation of natural CLA in dairy products is attributed to the increase in the availability 
of lipids by manipulation of feeds consisting mainly of grains and seed oils for ruminal biohydrogenation (Khanal and Olson, 2004). According to the study by Dhiman et al. (2000), feeding lipid sources, roasted cracked soybeans, soybean oil, and linseed oil, increased the CLA content of cattle milk when oils were accessible to the rumen microorganisms for biohydrogenation. Wang et al. (2005) reported that the amount of CLA was increased by the addition of fish oil, without causing an adverse effect on ruminal fermentation. In addition, Rana et al. (2012) showed that the effect of tanniniferous Terminalia chebula extract on the rumen biohydrogenation contributed to increased CLA content in the muscle of goat kids. On the other hand, B. fibrisolvens, which is known to be a major CLA-producing ruminal bacterium, exhibits high biohydrogenation activity, which consumes CLA. Therefore, the protection of CLA from the biohydrogenation attracted great attention. However, limited studies have shown on the stability of nano-encapsulated CLA against the ruminal biohydrogenation from by ruminal microbes.

This study aimed to evaluate the in vitro effect of chemically synthesized nano-encapsulated CLA on the stability of CLA and the ruminal fermentation characteristics.

\section{MATERIALS AND METHODS}

\section{Ruminal inoculum and in vitro incubation}

A fistulated Holstein cow was used as a donor of rumen fluid. The commercial concentrate (Saesam, Daehan feed Co., LTD., Incheon, Korea) was fed at $3 \mathrm{~kg}$ a day. Rice straw, water and mineral-vitamin block were allowed ad libitum. The rumen samples were collected before feeding. Rumen fluid was strained through four layers of cheesecloth before mixing with buffer and was maintained at $39^{\circ} \mathrm{C}$. The $30 \mathrm{~mL}$ of rumen fluid-buffer mixture, comprising McDougall buffer (McDougall, 1948) and rumen fluid in the ratio of 4 to 1 , was dispensed anaerobically into serum bottles containing $0.3 \mathrm{~g}$ of alfalfa substrate and additives $10 \%$ of substrate dry matter (DM), (free fatty acid form of CLA [CLA-FFA], nano-encapsulated CLA-FFA, triglyceride form of CLA [CLA-TG], nano-encapsulated CLA-TG). The serum bottles were filled with $\mathrm{O}_{2}$-free $\mathrm{CO}_{2}$ gas, and then capped with a rubber stopper. The bottles were kept in an incubator (JSGI-250T, JSR, Gongju, Korea) at $39^{\circ} \mathrm{C}$ for $6 \mathrm{~h}, 12 \mathrm{~h}, 24 \mathrm{~h}$, and $48 \mathrm{~h}$.

\section{Measurement and analysis}

Incubation was terminated by taking the serum bottles from the incubator at the indicated times of $6 \mathrm{~h}, 12 \mathrm{~h}, 24 \mathrm{~h}$, and $48 \mathrm{~h}$. Total gas production from serum bottles at the end of each incubation period was measured through the three- way stopcock using a $50 \mathrm{~mL}$ glass syringe connected to a needle. The $\mathrm{pH}$ was measured by $\mathrm{pH}$ meter (MP-220K, Mettler-Toledo, Columbus, $\mathrm{OH}$, USA). The supernatant and incubated mixture were transferred into a test tube and frozen at $-20^{\circ} \mathrm{C}$ for further analysis of total volatile fatty acids (tVFAs), fatty acids and rumen microbial populations, respectively.

Ethylation for fatty acid analysis: A total of $1 \mathrm{~mL}$ of medium to which $1 \mathrm{mg}$ of heptadecanoic acid $\left(\mathrm{C}_{17: 0}\right)$ (H3500, Sigma, St. Louis, MO, USA) was added as an internal standard (IS) was extracted with $12 \mathrm{~mL}$ chloroform/methanol $(1: 1, \mathrm{v} / \mathrm{v})$. The lower layer was mixed vigorously with $2 \mathrm{~mL}$ of $0.88 \% \mathrm{KCl}$ solution and then evaporated with nitrogen until dry. The extracted lipids were ethylated using $10 \mathrm{~mL}$ of $2 \% \mathrm{H}_{2} \mathrm{SO}_{4}$ in ethanol at $80^{\circ} \mathrm{C}$ for $60 \mathrm{~min}$ (Kim et al., 2001). After addition of $8 \mathrm{~mL}$ of saturated $\mathrm{NaCl}$ solution and $4 \mathrm{~mL}$ of $\mathrm{n}$-hexane, fatty acid ethyl esters were obtained in the n-hexane layer; they were analyzed for total fatty acids, including CLA isomers, using a 7890A gas chromatograph with a flame ionization detector (Agilent Technologies, Wilmington, DE, USA). The fatty acid ethyl esters were separated using a Supelcowax-10-fused silica capillary column $(100 \mathrm{~m} \times 0.25$ $\mathrm{mm}$ inner diameter, $0.2 \mu \mathrm{m}$ film thickness; Supelco, Inc., Bellefonte, PA, USA) with $1.2 \mathrm{~mL} / \mathrm{min}$ of helium flow. The oven temperature was increased from $190^{\circ} \mathrm{C}$ to $240^{\circ} \mathrm{C}$ at the rate of $4^{\circ} \mathrm{C} / \mathrm{min}$. The temperature of both the injector and detector temperature was $260^{\circ} \mathrm{C}$. In total, $1 \mu \mathrm{L}$ of sample was injected into the column in the split mode $(50: 1)$. The peaks for each CLA isomer and other fatty acids were identified and quantified by comparing with the retention time and peak area of each fatty acid standard, respectively. IS was included as an internal reference before the extraction to determine the recovery of the fatty acids in each sample.

Potential gas production curves: The gas production profiles in triplicate were fitted to the following equation using a non-linear procedure (McDonald, 1981):

$$
Y=A\left(1-e^{-c(t-L)}\right)
$$

where $Y$ is the volume of gas production $(\mathrm{mL}$ per 100 mg DM) at time $t, A$ is gas production from soluble and insoluble fraction, $c$ is the rate of gas production, $L$ the lag time (h) and $t$ is the incubation time (h).

Volatile fatty acids (VFAs): The sample was centrifuged at $13,500 \times \mathrm{g}$ for $20 \mathrm{~min}$, and the supernatant was used to determine the concentrations of VFA in the sample. The HPLC (L-2200, Hitachi, Tokyo, Japan) fitted with a UV detector (L-2400, Hitachi, Japan) and a silica gel column (Metacarb 87H, Varian, CA, USA) was used to measure VFA contents as described by Muck and Dickerson (1988).

DNA extraction: A high-speed tissue homogenizer 
(Precellys 24, Bertin Crop., Rockville, MD, USA) which retains samples in screw-capped tubes containing silica beads was used for DNA extraction. Total nucleic acid was extracted from the incubated rumen samples by using the modified bead-beating protocol with the Soil kit (NucleoSpin Soil, Macherey-nagel Inc., Bethlehem, PA, USA). Nucleic acid concentrations were measured using a NanoDrop Spectrophotometer (ND-1000, Thermo Scientific, Wilmington, DE, USA).

Polymerase chain reaction primers: The polymerase chain reaction (PCR) primer sets used for amplification of Fibrobacter succinogenes ( $F$. succinogenes) (ForwardGGTATGGG ATGAGCTTGC, ReverseGCCTGCCCCTGAACTATC) and Butyrivibrio fibrisolvens (Forward-TAACATGAGAGT TTGATCCTGGCTC, Reverse-CGTTACTCACCCGTCCGC) were the same as those referenced by Tajima et al. (2001) and Yang et al. (2009), respectively. Total bacteria primer set (ForwardCGGCAACGAGCGCAACCC,

ReverseCCATTGTAGCACGTGTGTAGCC) was used as the internal standard (Demman and McSweeney, 2006).

Real-time PCR: Real-time PCR (RT-PCR) assays for enumeration of microbes were performed according to the methods described by Denman and McSweeney (2006) and Denman et al. (2007) on a real-time PCR Machine (7500 Real-Time PCR System, ABI, Foster City, CA, USA) using the SYBR Green kit $(2 \times$ Mastermix w/Low-Rox kit, m.biotech, Hanam, Korea). The values of cycle threshold (Ct) after PCR reactions were used to determine the fold change (number of fold difference) of different microbial populations relative to control without additives. The abundance of these microbes was expressed by the equation: relative quantification $=2^{-\Delta \mathrm{Ct}(\text { Target })-\Delta \mathrm{Ct}(\text { Control) }}$, where $\mathrm{Ct}$ represents the threshold cycle. All RT-PCR reaction mixtures (final volume, $20 \mu \mathrm{L}$ ) contained forward and reverse primers, the SYBR Green Supermix and DNA template $(100 \mathrm{ng})$. A negative control without the template DNA was used in every RT-PCR assay for each primer. The RT-PCR amplification of the target DNA, including the annealing and the extension temperatures, was performed as previously described (Tajima et al., 2001; Yang et al., 2009).

\section{Statistical analysis}

All experiments were performed in triplicate, and data were expressed as means \pm standard deviations. Statistical analyses were performed by analysis of variation. The effects of nano-encapsulation on total gas production, $\mathrm{pH}$ and tVFA concentration were compared with the controls and significant differences between treatment means were examined using Duncan's multiple comparison tests. A $\mathrm{p}<0.05$ was considered to be statistically significant ( $R$, version 3.2.0, 2015).

\section{RESULTS AND DISCUSSION}

\section{Biohydrogenation with nano-encapsulated CLA by Butyrivibrio fibrisolvens}

The cis-9, trans-11 CLA isomer is an intermediate in the biohydrogenation of LA to stearic acid (SA) by the anaerobic rumen bacterium B. fibrisolvens (Kepler et al., 1966). The conversion sequence of LA to SA involves at least three steps. The sequence begins with the isomerization of LA to cis-9, trans-11 CLA, followed by the hydrogenation of the cis-double bond of the conjugated diene to yield a trans-11 octadecenoic acid (trans-vaccenic acid). In addition, VA can also be converted to cis-9, trans11 CLA by the $\Delta^{9}$-desaturase enzyme in mammary tissue, providing another mechanism for CLA formation in milk (Griinari and Bauman, 1999). This in vitro trial showed the protective effect of nano-encapsulated CLAs against the biohydrogenation activity of $B$. fibrisolvens (Figure 1). The concentration of cis-9, trans-11 CLA was decreased, and that of VA was increased by CLA-FFA at the initial incubation time (Figure 1a). Thereafter, no further decrease in the concentration of cis-9, trans-11 CLA was observed despite prolonged incubation. On the other hand, the concentration slightly was decreased by biohydrogenation when $B$. fibrisolvens was incubated with nano-encapsulated CLA-FFA (Figure 1b). These findings showed that $B$. fibrisolvens had a high degree of biohydrogenation activity and low CLA-producing activity when incubated with CLA-FFA, and that CLA-FFA might be protected from the biohydrogenation acitivity of $B$. fibrisolvens by nanoencapsulation. In the group of $B$. fibrisolvens incubated with CLA-TG, cis-9, trans-11 CLA, and VA concentrations were decreased, while the concentrations further increased after incubation with nano-encapsulated (Figure 1c, 1d). This finding showed that the nano-encapsulated CLA-TG was less protective against the biohydrogenation activity of B. fibrisolvens compared with CLA-TG. The CLA protection rates of CLA-FFA, nano-encapsulated CLA-FFA, CLA-TG, and nano-encapsulated CLA-TG after $48 \mathrm{~h}$ of incubation were $36 \%, 80 \%, 87 \%$, and $73 \%$, respectively. Therefore, nano-encapsulation was more effective against the in vitro biohydrogenation activity of $B$. fibrisolvens when incubated with CLA-FFA rather than CLA-TG. Nano-encapsulation, thus, might be used as an alternative technique to protect target materials against ruminal biohydrogenation in further studies.

\section{In vitro ruminal fermentation characteristics with nano- encapsulated CLA}

The in vitro ruminal fermentation profiles are shown in Table 1. The change in ruminal $\mathrm{pH}$ is an important factor not only for the amount of intermediates during biohydrogenation of LA but also for the microbial 
(a) CLA-FFA

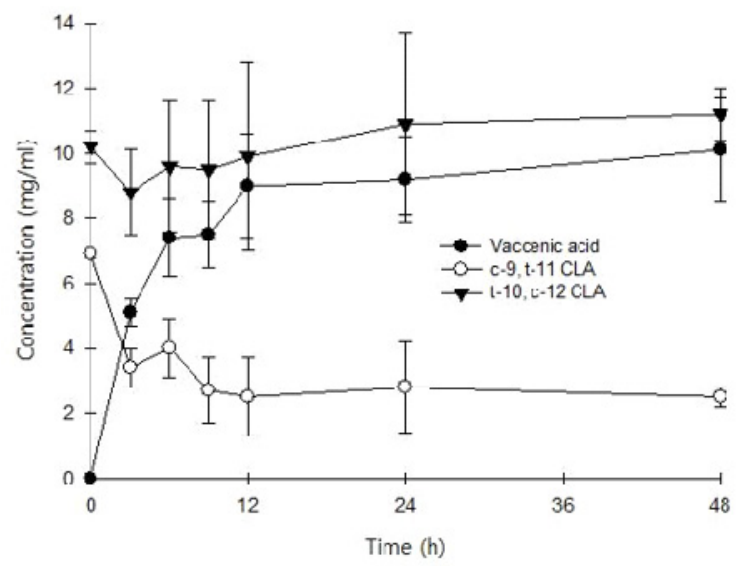

(c) CLA-TG

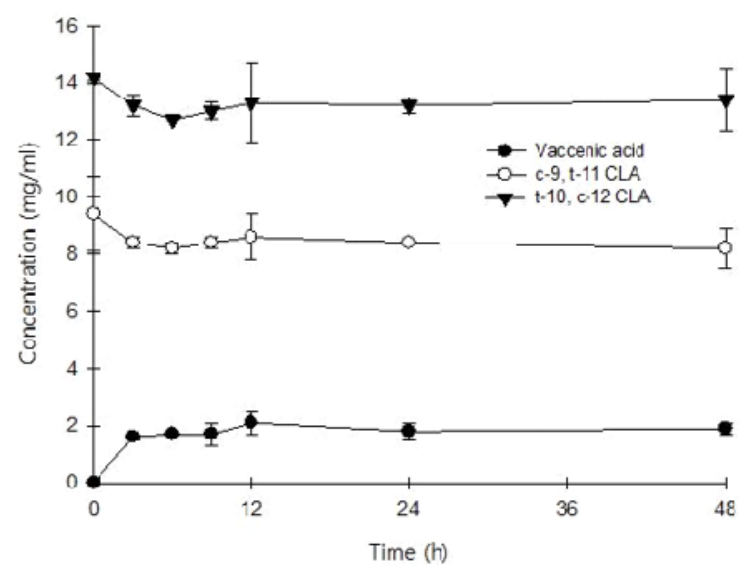

(b) Nano-encapsulated CLA-FFA

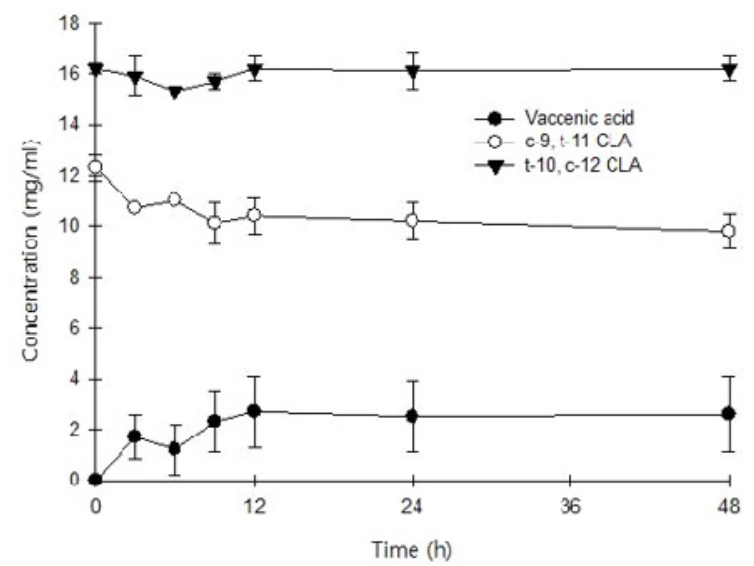

(d) Nano-encapsulated CLA-TG

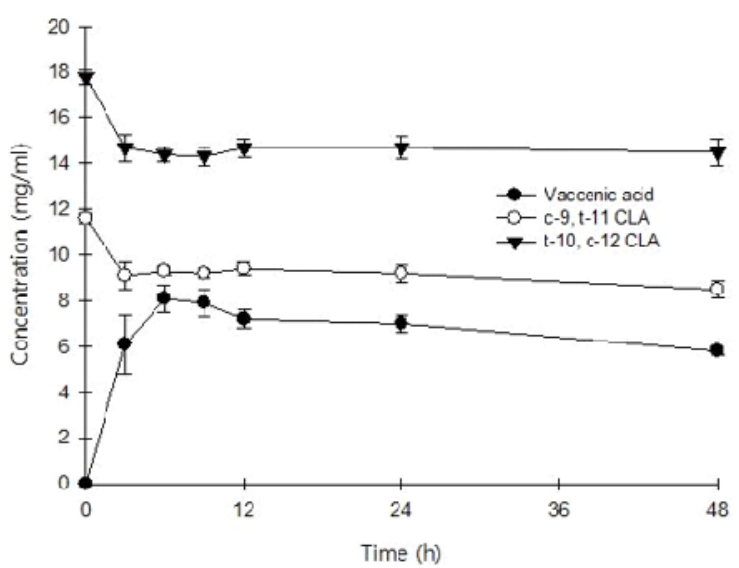

Figure 1. The in vitro protection effects of conjugated linoleic acids (CLAs) against B. fibrisolvens. (a), free fatty acid form of CLA (CLA-FFA); (b) nano-encapsulated CLA-FFA; (c) triglyceride form of CLA (CLA-TG); (d) nano-encapsulated CLA-TG.

Table 1. The effects of conjugated linoleic acids (CLAs) on ruminal fermentation characteristics for $48 \mathrm{~h}$ incubation

\begin{tabular}{|c|c|c|c|c|c|c|}
\hline \multirow{2}{*}{ Incubation time } & \multirow{2}{*}{ Control } & \multicolumn{4}{|c|}{ Treatments } & \multirow{2}{*}{ SEM } \\
\hline & & $\mathrm{F}$ & NF & $\mathrm{T}$ & NT & \\
\hline & \multicolumn{6}{|c|}{ 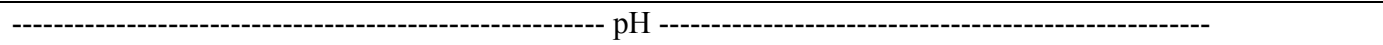 } \\
\hline $6 \mathrm{~h}$ & $6.78^{\mathrm{a}}$ & $6.71^{\mathrm{ab}}$ & $6.69^{\mathrm{b}}$ & $6.72^{\mathrm{ab}}$ & $6.67^{\mathrm{b}}$ & 0.026 \\
\hline $12 \mathrm{~h}$ & $6.70^{\mathrm{bc}}$ & $6.76^{\mathrm{a}}$ & $6.67^{\mathrm{c}}$ & $6.72^{\mathrm{ab}}$ & $6.69^{\mathrm{bc}}$ & 0.016 \\
\hline $24 \mathrm{~h}$ & $6.68^{\mathrm{a}}$ & $6.60^{\mathrm{ab}}$ & $6.28^{\mathrm{c}}$ & $6.55^{\mathrm{b}}$ & $6.25^{\mathrm{c}}$ & 0.026 \\
\hline $48 \mathrm{~h}$ & $6.64^{\mathrm{a}}$ & $6.51^{\mathrm{b}}$ & $6.01^{\mathrm{c}}$ & $6.51^{\mathrm{b}}$ & $5.96^{\mathrm{c}}$ & 0.025 \\
\hline $6 \mathrm{~h}$ & $36.67^{\mathrm{a}}$ & $28.87^{\mathrm{b}}$ & $\begin{array}{c}\text { oduction } \\
38.90^{\mathrm{a}}\end{array}$ & $38.90^{\mathrm{a}}$ & $37.77^{\mathrm{a}}$ & 1.990 \\
\hline $12 \mathrm{~h}$ & $74.43^{\mathrm{a}}$ & $44.43^{c}$ & $70.00^{\mathrm{a}}$ & $72.20^{\mathrm{a}}$ & $58.90^{\mathrm{b}}$ & 2.488 \\
\hline $24 \mathrm{~h}$ & $138.90^{\mathrm{c}}$ & $98.87^{\mathrm{d}}$ & $172.23^{\mathrm{a}}$ & $136.67^{c}$ & $159.43^{\mathrm{b}}$ & 3.991 \\
\hline $48 \mathrm{~h}$ & $162.23^{b}$ & $112.77^{\mathrm{c}}$ & $225.00^{\mathrm{a}}$ & $145.53^{\mathrm{b}}$ & $213.33^{\mathrm{a}}$ & 6.046 \\
\hline $6 \mathrm{~h}$ & $20.21^{\mathrm{a}}$ & $12.72^{\mathrm{c}}$ & $\begin{array}{l}- \text { tVFAs } \\
14.45^{\mathrm{bc}}\end{array}$ & $16.64^{b}$ & $11.93^{\mathrm{c}}$ & 1.099 \\
\hline $12 \mathrm{~h}$ & $25.54^{\mathrm{a}}$ & $20.37^{\mathrm{b}}$ & $21.40^{\mathrm{b}}$ & $24.86^{\mathrm{a}}$ & $21.06^{\mathrm{b}}$ & 0.555 \\
\hline $24 \mathrm{~h}$ & $35.20^{\mathrm{b}}$ & $32.74^{\mathrm{b}}$ & $45.88^{\mathrm{a}}$ & $35.07^{\mathrm{b}}$ & $46.61^{\mathrm{a}}$ & 0.974 \\
\hline $48 \mathrm{~h}$ & $40.93^{c}$ & $37.48^{\mathrm{d}}$ & $61.76^{\mathrm{b}}$ & $38.05^{\mathrm{cd}}$ & $67.15^{\mathrm{a}}$ & 0.942 \\
\hline
\end{tabular}

Control, none addition; F, free fatty acid form of CLA (CLA-FFA); NF, nano-encapsulated CLA-FFA; T, triglyceride form of CLA (CLA-TG); NT, nanoencapsulated CLA-TG; SEM, standard error of the mean; tVFAs, total volatile fatty acids.

${ }^{a-d}$ Means in the same row with different superscripts differ significantly $(\mathrm{p}<0.05)$. 
Table 2. The effects of conjugated linoleic acids (CLAs) on the potential gas production, specific rates of digestion and lag phase

\begin{tabular}{lccccccc}
\hline \multirow{2}{*}{ Item } & \multirow{2}{*}{ Control } & \multicolumn{4}{c}{ Treatments } & \multirow{2}{*}{ SEM } & \multirow{2}{*}{ p-value } \\
\cline { 3 - 6 } & & $\mathrm{F}$ & $\mathrm{NF}$ & $\mathrm{T}$ & $\mathrm{NT}$ & & \\
\hline Gas production (mL per 100 mg DM) & $177.67^{\mathrm{b}}$ & $124.03^{\mathrm{c}}$ & $271.03^{\mathrm{a}}$ & $154.23^{\mathrm{bc}}$ & $263.27^{\mathrm{a}}$ & 14.475 & $<0.001$ \\
Specific rates of digestion (per h) & $0.070^{\mathrm{ab}}$ & $0.061^{\mathrm{ac}}$ & $0.039^{\mathrm{c}}$ & $0.083^{\mathrm{a}}$ & $0.045^{\mathrm{bc}}$ & 0.009 & 0.0319 \\
Lag phase (h) & 2.57 & 2.28 & 2.92 & 2.80 & 3.20 & 0.573 & $\mathrm{NS}$ \\
$\mathrm{R}^{2}$ & 0.95 & 0.86 & 0.91 & 0.89 & 0.93 & & \\
\hline
\end{tabular}

Control, none addition; F, free fatty acid form of CLA (CLA-FFA); NF, nano-encapsulated CLA-FFA; T, triglyceride form of CLA (CLA-TG); NT, nanoencapsulated CLA-TG; SEM, standard error of the mean; NS, not significantly.

${ }^{a-c}$ Means in the same row with different superscripts differ significantly $(p<0.05)$.

enzymatic activity in CLA production. Lee (2013) reported that the concentration of trans-11 $\mathrm{C}_{18: 1}$ was increased more at $\mathrm{pH} 6.5$ than at $\mathrm{pH}$ 5.5. In this in vitro ruminal fermentation, the $\mathrm{pH}$ was decreased by the addition of nanoencapsulated CLA-FFA and CLA-TG at $24 \mathrm{~h}$ incubation. The total gas production was significantly increased after 24 $\mathrm{h}$ incubation when nano-encapsulated CLA was added $(p<0.05)$. The potential in vitro ruminal fermentation and gas production curves are shown in Table 2 and Figure 2, respectively. Nano-encapsulated CLA-FFA and CLA-TG showed the higher potential for the ruminal fermentation than the control and non-nano-encapsulated CLA-FFA and CLA-TG. The tVFA concentration significantly was increased by nano-encapsulated CLA-FFA and CLA-TG compared with the control as well as CLA-FFA and CLATG after $24 \mathrm{~h}$ incubation. The effect of CLAs on the reduction rate of CLA in in vitro ruminal fermentation is shown in Figure 3. The reduction rates of CLA concentration with CLAs (CLA-FFA, nano-encapsulated CLA-FFA, CLA-TG, and nano-encapsulated CLA-TG) were $23.9 \%, 30.9 \%, 31.1 \%$, and $22.3 \%$ during the initial 12 $\mathrm{h}$ incubation, while they were $22.3 \%, 15.7 \%, 18.8 \%$, and $27.0 \%$ during 12 to $24 \mathrm{~h}$ incubation, respectively. Although the rates of decrease in CLA concentration on incubation with CLA-FFA and nano-encapsulated CLA-TG were low

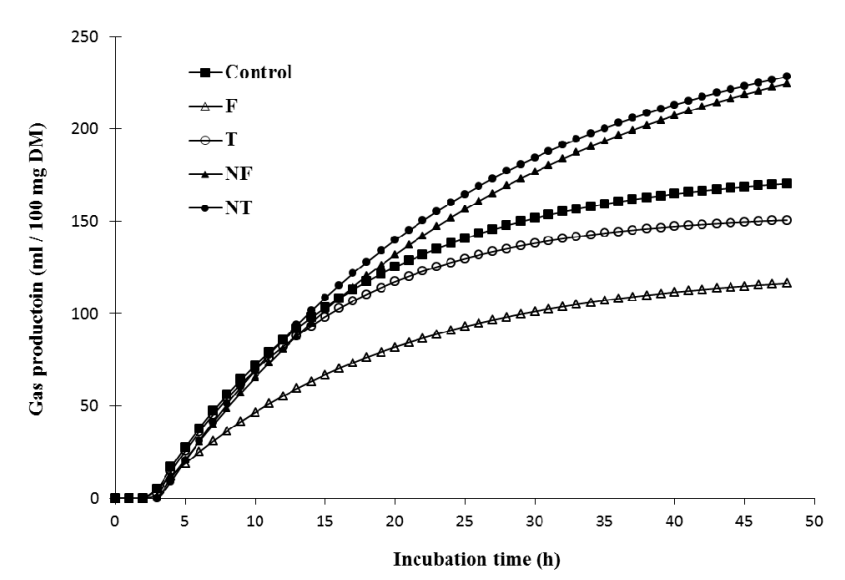

Figure 2. The potential gas production curves from the in vitro fermentation by addition of CLAs. F, free fatty acid form of CLA (CLA-FFA); NF, nano-encapsulated CLA-FFA; T, triglyceride form of CLA (CLA-TG); NT, nano-encapsulated CLA-TG. at the initial incubation times, they were increased with the increase in incubation time. The total gas production and tVFAs concentration also were decreased by the addition of CLA-FFA and nano-encapsulated CLA-TG at the initial incubation times. The rate of decrease in CLA concentration when incubated with the nano-encapsulated CLA-FFA was the lowest from 12 to $24 \mathrm{~h}$ of incubation compared with the rates when other types of CLAs were added. These findings showed that nano-encapsulated CLA-FFA might in part influence the ruminal fermentation characteristics without adversely affecting incubation process, and might have a protective effect on ruminal biohydrogenation. The population change of $F$. succinogenes and B. fibrisolvens is shown in Figure 4. The population of $F$. succinogenes was increased on incubation with nano-encapsulated CLA-FFA, while that of $B$. fibrisolvens was decreased in the in vitro ruminal fermentation (Figure 4). According to the study of Jenkins et al. (2008), bacteria play the important role in fatty acid biohydrogenation. B. fibrisolvens is the active bacteria isolated from the rumen as being most involved in biohydrogenation (Paillard et al., 2007a; Lourenço et al., 2010). The previous studies also reported that $B$.

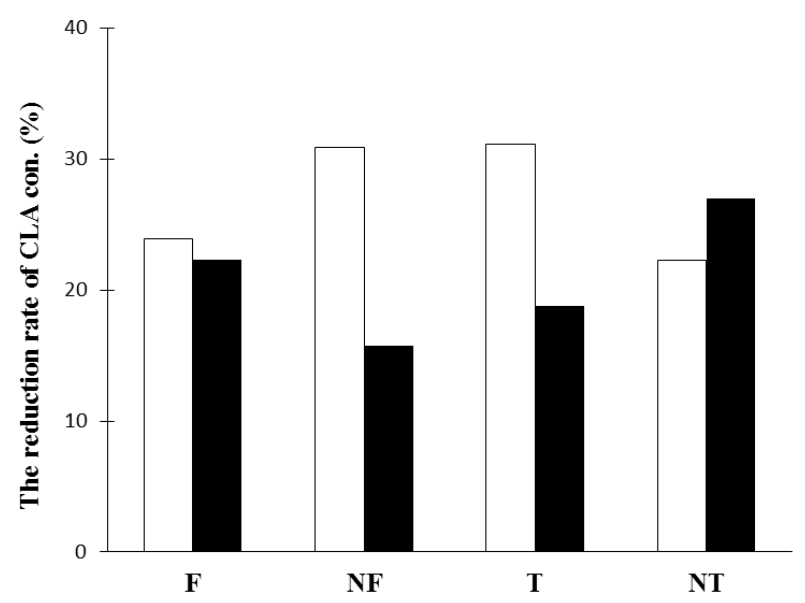

Figure 3. The reduction rate of conjugated linoleic acids (CLA) concentration (\%) in in vitro ruminal fermentation by the addition of CLAs. $\square$, from 0 to $12 \mathrm{~h}$ of incubation; $\mathbf{\square}$, from 12 to $24 \mathrm{~h}$ of incubation; F, free fatty acid form of CLA (CLA-FFA); NF, nanoencapsulated CLA-FFA; T, triglyceride form of CLA (CLA-TG); NT, nano-encapsulated CLA-TG. 

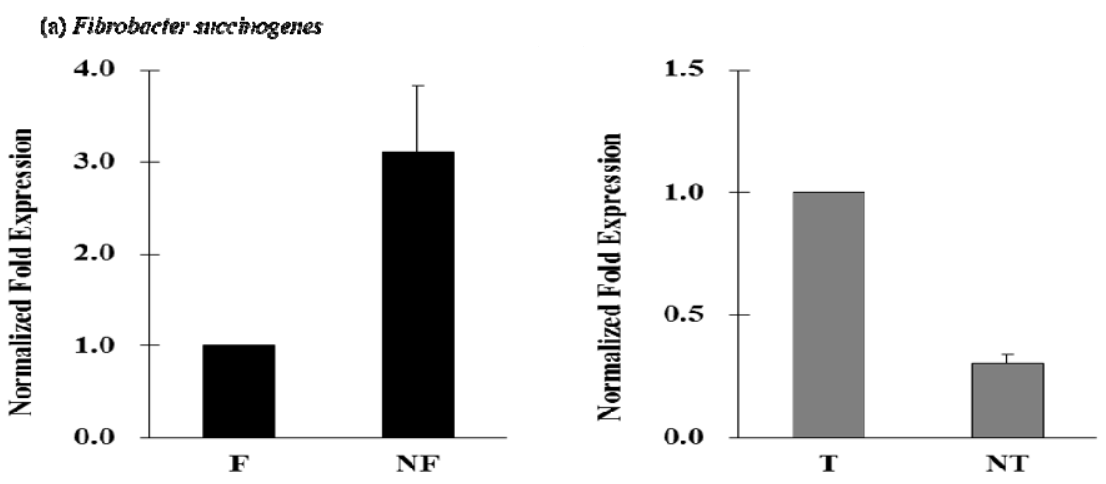

(b) Buthrinibrio fibrisodress
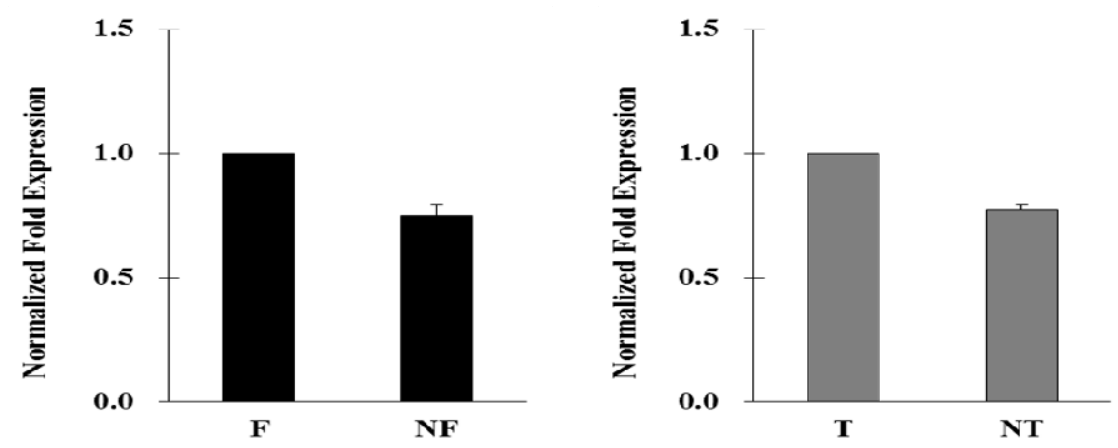

Figure 4. Relative quantification analysis of microbes in in vitro ruminal fermentation by the addition of conjugated linoleic acids (CLAs) after $24 \mathrm{~h}$ incubation. F, free fatty acid form of CLA (CLA-FFA); NF, nano-encapsulated CLA-FFA; T, triglyceride form of CLA (CLA-TG); NT, nano-encapsulated CLA-TG.

fibrisolvens was identified to form CLA and VA as intermediates during the biohydrogenation of LA (Polan et al., 1964; Kepler et al., 1966). The nano-encapsulation might influence in the biohydrogenation pathways which is converted CLA to VA in this in vitro study, and thus the nano-encapsulated CLAs might lead to decrease in the $B$. fibrisolvens population.

In conclusion, protection from biohydrogenation and stability of CLA afforded by nano-encapsulation were examined under artificial rumen conditions to characterize the nano-encapsulated CLAs. We observed that the nanoencapsulated CLA-FFA was the most effective against the in vitro ruminal biohydrogenation without adversely affecting ruminal fermentation. Although these in vitro results do not completely explain the in vivo rumen ecosystem, nano-encapsulation could be applied to enhance CLA levels by increasing the stability of CLA in the ruminal ecosystem to enrich CLA in dairy products without adversely affecting ruminal fermentation. However this method should be further optimized by exploring other unknown environmental factors. An in vivo ruminal study is needed to better understand the biohydrogenation pathways of nano-encapsulated CLAs in ruminants.

\section{CONFLICT OF INTEREST}

We certify that there is no conflict of interest with any financial organization regarding the material discussed in the manuscript.

\section{ACKNOWLEDGMENTS}

This research was supported by High Value-added Food Technology Development Program, Ministry of Agriculture, Food and Rural Affairs(MAFRA).

E. T. Kim was supported by Postdoctoral Fellowship Program of National Institute of Animal Science, Rural Development Administration, Republic of Korea.

\section{REFERENCES}

Chin, S. F., W. Liu, J. M. Storkson, Y. L. Ha, and M. W. 1992. Pariza. Dietary sources of conjugated dienoic isomers of linoleic acid, a newly recognized class of anticarcinogens. J. Food Compost. Anal. 5:185-197.

Cook, M. E., D. Butz, G. M. Li, M. Pariza, L. Whigham, and M. Yang. 2003. Conjugated linoleic acid enhances immune responses but protects against the collateral damage of immune events. In Advances in Conjugated Linoleic Acid Research (Eds. J. L. Sébédio, W. W. Christie, and R. Adlof). Vol. 2, pp. 283-291. AOCS Press, Champaign, IL, USA.

Crumb, D. J. 2011. Conjugated linoleic acid (CLA)-An Overview. Int. J. Appl. Res. Nat. Prod. 4:12-18.

Denman, S. E. and C. S. McSweeney. 2006. Development of a real-time PCR assay for monitoring anaerobic fungal and 
cellulolytic bacterial populations within the rumen. FEMS Microbiol. Ecol. 58:572-582.

Denman, S. E., N. W. Tomkins, and C. S. McSweeney. 2007. Quantitation and diversity analysis of ruminal methanogenic populations in response to the antimethanogenic compound bromochloromethane. FEMS Microbiol. Ecol. 62:313-322.

Dhiman, T. R., L. D. Satter, M. W. Pariza, M. P. Galli, K. Albright, and M. X. Tolosa. 2000. Conjugated linoleic acid (CLA) content of milk from cows offered diets rich in linoleic and linolenic acid. J. Dairy Sci. 83:1016-1027.

Dilzer, A. and Y. Park. 2012. Implication of conjugated linoleic acid (CLA) in human health. Crit. Rev. Food Sci. 52:488-513.

Griinari, J. M. and D. E. Bauman. 1999. Biosynthesis of conjugated linoleic acid and its incorporation into meat and milk in ruminants. In advances in conjugated linoleic acid research. Vol. 1. (Eds. M. P. Yurawecz, M. M. Mossoba, J. K. G. Kramer, M. W. Pariza, and G. J. Nelson) p. 180-200. AOCS Press, Champaign, IL, USA.

Jenkins, T. C., R. J. Wallace, P. J. Moate, and E. E. Mosley. 2008. Recent advances in biohydrogenation of unsaturated fatty acids within the rumen microbial ecosystem. J. Anim. Sci. 86:397-412.

Kathirvelan, C., A. K. Tyagi, and P. Krishnamurthi. 2008. Influence of conjugated Linoleic acid ghee feeding on cancer incidences and histopathological changes in 7,12 dimethlybenz (a) anthrazene induced mammary glandcarcenogenesis in rats. Veterinarski Arhiv. 78:511-520.

Kepler, C. R., K. P. Hirons, J. J. Mcneill, and S. S. Tove. 1966. Intermediates and products of the biohydrogenation of linoleic acid by Butyrivibrio fibrisolvens. J. Biol. Chem. 241:13501354.

Khanal, R. C. and K. C. Olson. 2004. Factors affecting conjugated linoleic acid (CLA) content in milk, meat, and egg: A review. Pakistan J. Nutr. 3:82-98.

Kim, I. H., C. S. Kim, S. H. Cho, K. W. Lee, S. H. Chung, and B. S. Tae. 2001. Lipase-catalyzed incorporation of conjugated linoleic acid into tricaprylin. J. Am. Oil Chem. Soc. 78:547551.

Kritchevsky, D. 2000. Antimutagenic and some other effects of conjugated linoleic acid. Br. J. Nutr. 83:459-465.

Lee, Y. -J. 2013. Effect of pH on conjugated linoleic acid (CLA) formation of linolenic acid biohydrogenation by ruminal microorganisms. J. Microbiol. 51:471-476.

Lourenço, M., E. Ramos-Morales, and R. J. Wallace. 2010. The role of microbes in rumen lipolysis and biohydrogenation and their manipulation. Animal 4:1008-1023.
McDonald, I. 1981. A revised model for the estimation of protein degradability in the rumen. J. Agric. Sci. 96:251-252.

McDougall, E. I. 1948. Studies on ruminant saliva. I. The composition and output of sheep's saliva. Biochem. J. 43:99109.

Muck, R. E. and J. T. Dickerson. 1988. Storage temperature effects on proteolysis in alfalfa silage. Trans. ASAE. 31:1005-1009.

Paillard, D., N. McKain, L. C. Chaudhary, N. D. Walker, F. Pizette, I. Koppova, N. R. McEwan, J. Kopecny, P. E. Vercoe, P. Louis, and R. J. Wallace. 2007a. Relation between phylogenetic position, lipid metabolism and butyrate production by different Butyrivibrio-like bacteria from the rumen. Antonie. Van Leeuwenhoek. 91:417-422.

Parodi, P. W. 2003. Conjugated linoleic acid in food. In advances in conjugated linoleic acid research. Vol. 2. (Ed. J. Sebedio, W. W. Christie, and R. Adlof) p. 101-121. AOCS Press, Champaign, IL, USA.

Polan, C. E., J. J. McNeill, and S. B. Tove. 1964. Biohydrogenation of unsaturated fatty acids by rumen bacteria. J. Bacteriol. 88:1056-1064.

R. R development core team. 2015. A language and environment for statistical computing. $\mathrm{R}$ foundation for statistical computing, Vienna, Austria. http://www.r-project.org.

Rana, M. S., A. Tyagi, S. K. Asraf Hossain, and A. K. Tyagi. 2012. Effect of tanniniferous Terminalia chebula extract on rumen biohydrogenation, $\Delta^{9}$-desaturase activity, CLA content and fatty acid composition in longissimus dorsi muscle of kids. Meat Sci. 90:558-563.

Shantha, N. C., A. D. Crum, and E. A. Decker. 1994. Evaluation of conjugated linoleic acid concentrations in cooked beef. J. Agric. Food Chem. 42:1757-1760.

Tajima, K., R. I. Aminov, T. Nagamine, H. Matsui, M. Nakamura, and Y. Benno. 2001. Diet-dependent shifts in the bacterial population of the rumen revealed with real-time PCR. Appl. Environ. Microbiol. 67:2766-2774.

Wang, J. H., S. H. Choi, C. G. Yan, and M. K. Song. 2005. Effect of monensin and fish oil supplementation on biohydrogenation and CLA production by rumen bacteria in vitro when incubated with safflower oil. Asian Australas. J. Anim. Sci. 18:221-225.

Yang, S. L., D. P. Bu, J. Q. Wang, Z. Y. Hu, D. Li, H. Y. Wei, L. Y. Zhou, and J. J. Loor. 2009. Soybean oil and linseed oil supplementation affect profiles of ruminal microorganisms in dairy cows. Animal 3:1562-1569. 